\title{
Electric and magnetic fields of the brain accompanying internal simulation of movement
}

\author{
Wilfried Lang ${ }^{\mathrm{a}, *}$, Douglas Cheyne ${ }^{\mathrm{b}}$, Peter Höllinger ${ }^{\mathrm{a}}$, Willi Gerschlager ${ }^{\mathrm{a}}$, \\ Gerald Lindinger ${ }^{a}$ \\ ${ }^{a}$ University Clinic of Neurology Vienna, Währinger Gürtel 18-20, A-1090 Vienna, Austria \\ ${ }^{\mathrm{b}}$ Brain Behaviour Laboratory, School of Kinesiology, Simon Fraser University, Burnaby, B.C., Canada V5A 1 S6
}

Accepted 22 August 1995

\begin{abstract}
Methods of functional brain imaging have been used to identify brain structures which are active during internal simulation of movements (ISM). Between 1977 and 1993 it was consistently reported that the primary motor cortex (MI) is not active during ISM whereas other cortical areas, in particular the supplementary motor area (SMA) are active. ISM was assumed to be a situation of 'internal programming'. Brain systems involved in ISM or 'programming' were hypothesized to be superior to and separable from 'executive system' including MI. We have studied electric and magnetic fields of the brain when subjects internally simulated either a single movement or a sequence of movements. Results of the studies are consistent with the assumption that MI is active with ISM. Internally subjects experienced effort which was required to inhibit overt movements during ISM. A recent EEG study showed different patterns of cortical activity with ISM and with movement inhibition suggesting that different brain structures may be active during ISM and movement inhibition [23].
\end{abstract}

Keywords: Motor imagery; Motor Cortex; Primary motor cortex; Magnetoencephalography

\section{Introduction}

When we experience the ability to imagine or 'internally simulate' a movement with its temporal and spatial sequencing we produce internal sensory images that resemble those which would arise when actually executing the movement. Furthermore, we experience effort to prevent overt movements during the performance of what we will refer to as the 'internal simulation of movement' (ISM). ISM was performed in the absence of overt movements. Subthreshold, task-specific muscular activity during ISM has been reported in some studies $[17,34]$ but not in others (e.g. [27]).

Several attempts have been made to identify brain structures which are activated during ISM: Ingvar and Philipson [16] measured changes of regional cerebral blood flow (rCBF) when subjects either executed rhythmic clenching movements of the right hand or imagined to do so. 'Motor ideation' as it was termed by these investiga-

\footnotetext{
* Corresponding author. Fax: (43) (1) 40400/3141.
}

tors, caused increased rCBF in frontal and temporal areas but no changes in the rolandic area. The absence of MI-activity was taken as evidence that imagination of movements constitutes a state of 'pure mentation'. When subjects executed rhythmic movements blood flow increased mainly in the rolandic area. Roland et al. [27] studied changes of $\mathrm{rCBF}$ when subjects either executed or 'internally simulated' a sequence of self-paced thumb to digits oppositions. rCBF was found to increase exclusively in the SMA during 'internal simulation'. During execution of the same motor sequence rCBF increased in both SMAs and in the MI which was contralateral to the performing hand. The state of internal simulation of a movement was conceived to reflect the process of 'internal programming'. It was suggested that "the supplementary motor areas are programming areas for motor subroutines and that these areas form a queue of time-ordered motor commands before voluntary movements are executed by way of the primary motor area (p. 118)'. Subsequent studies employed different methods (SPECT, PET, functional MRI) and various tasks of ISM. It was consistently reported that SMA is active with internal simulation of movements but 
MI is not active $[8,9,11,26,28,30]$. In addition to SMA other parts of the cortex were reported to be active with ISM although with little consistency, possibly due to differences among the tasks employed.

Based on these findings it has been hypothesized that (1) ISM is 'purely mental' since the motor executive system (MI and descending cortico-spinal pathways) is not active [16], and (2) ISM requires inhibition of overt movement which may be achieved by blocking information flowing from higher motor areas (e.g. SMA) to MI [7,27].

Observations on the rolandic (central) mu rhythm have not been taken into account in this discussion; The rolandic mu rhythm has been localized in precentral recordings in the electrocorticogram [14] and is strongly related to function of MI; Rolandic mu is blocked with active movements. This blocking effect has a somatotopic distribution $[2,12]$ and is elicited by mere thoughts about performing movements $[2,12,18]$. Blocking was even present when persons with amputations of the limbs imagined to perform movements with the phantom limb $[13,18]$. In the EEG the mu rhythm is desynchronized prior and during hand movements in a small scalp area overlying MI [25].

In this paper we describe two studies which support the view that MI is active with internal simulation of movements.

\section{EEG-studies}

Slow brain potentials (SPs) were recorded when subjects executed motor sequences of either hand or when they imagined to do so [1]. At locations above the MI-hand area (left MI: C3; right MI: C4) amplitudes of SPs systematically varied with the side executing the task: movements by the right hand caused an increase of negative SPs in C3. Conversely, movements by the left hand caused an increase of SPs in C4. These results were consistent with earlier studies $[10,21]$. Qualitatively the same and quantitatively similar effects of the side of the hand were present when subjects internally simulated the motor sequence. These observations support the concept of MI activation with ISM.
The distribution of SPs was different with task execution and imagination. Changes of SPs between these conditions were localized in central recordings $(\mathrm{C} 3, \mathrm{Cz}, \mathrm{C} 4)$ with larger amplitudes when executing the tasks than when imagining to do so. Proprioceptive and kinaesthetic input with task execution or an enhanced level of activation of the cortico-motoneural system with task execution may account for this difference [1].

\section{MEG-studies}

Magnetoencephalography (MEG) has been used in three tasks: (1) self-paced, flexions of the right index finger at irregular intervals between $4 \mathrm{~s}$ and $8 \mathrm{~s}$. (2) An omitted tone paradigm with tones $(1 \mathrm{kHz}, 60 \mathrm{~dB}, 50 \mathrm{~ms}$ in duration) being delivered at regular $1000 \mathrm{~ms}$ intervals to both ears via plastic tubes. Omissions occurred after every three tones. Subjects were instructed to flex the right index finger in time with the 'omitted tone'. (3) Omitted tone paradigm with the instruction to imagine to perform the movement in time with the omitted tone. In each condition 150 periods were averaged, the conditions were randomized in blocks of 50 trials. The MEG system consisted of 64, first-order axial gradiometers distributed over a helmet shaped dewar (CTF Systems Inc.).

Fig. 1, Fig. 2, Figs. 3 and 4 present results in one subject. With voluntary movements (task 1 ) the pattern of magnetic fields at movement onset can be explained by two current dipoles, one located in the hand region of MI in each hemisphere (Fig. 1). Location and orientation of each dipole are consistent with the assumption that they reflect intracellular, surface-to-depth current flow in the anterior bank of the central sulcus of each hemisphere (area 4; MI; for review: [20]). The two dipoles explained $92 \%$ of the variance of the observed data at movement onset. In task 2 , the subject performed brisk finger flexions in time with the omitted tone. Average movement onset was $800 \mathrm{~ms}$ after the preceding tone. Thus, average timing of the movement was $200 \mathrm{~ms}$ before the 'omitted tone'. The subject's timing of movement onset from trial to trial was sufficiently consistent to create a synchronized rise in

Fig. 1. Upper part: Pattern of magnetic fields at the onset of self-paced finger flexion. Positions where field lines exit the scalp are blue, positions where field lines enter the scalp are red. Lower part: $92 \%$ of the observed data at movement onset could be explained by the two dipoles in the primary motor cortex of either hemisphere. Dipole locations and directions of current flow are shown in different head projections.

Fig. 2. Upper part: Average movement onset was $800 \mathrm{~ms}$ after the preceding tone in the 'omitted stimulus paradigm' (EMG, electromyography of the M. flexor indicis right). Pattern of magnetic fields at the time of averaged movement onset (as in Fig. 1). 93\% of the observed data at movement onset could be explained by the two dipoles in the primary motor cortex (locations and directions of dipoles as in Fig. 1). Lower part: Pattern of magnetic fields at the time ( $800 \mathrm{~ms}$ after the preceding tone) when internal simulation of movement was assumed to take place.

Fig. 3. Upper part: Pattern of magnetic fields at the time of the averaged movement onset. Lower part: Pattern of magnetic fields at the time (896 ms after the preceding tone) when internal simulation of movement was supposed to take place.

Fig. 4. Left side: $\mathrm{N} 100 \mathrm{~m}$ following the presentation of the third tone. Right side: Pattern of magnetic fields when the subject imagined to hear the 'omitted tone'. 
the averaged and rectified EMG (Fig. 2; upper part). The pattern of magnetic fields at movement onset is displayed in Fig. 2 (upper part). Using the solution for dipole location and orientation for the same time point in task 1 , 93\% of data could be explained at average movement onset in task 2. Based on the assumption that the timing of the internally simulated movement is comparable to the timing of the executed movement, the pattern of magnetic fields was calculated $800 \mathrm{~ms}$ after the preceding tone in task 3. As can be seen in Fig. 2 (lower part) the same
Fig. 1

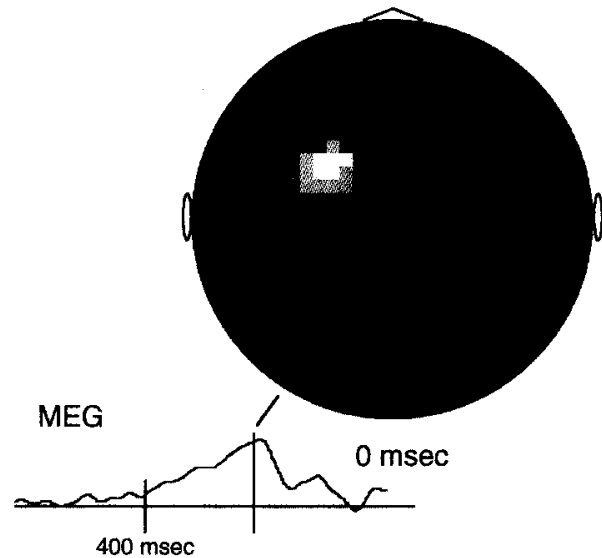

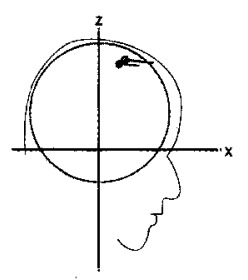

sagittal

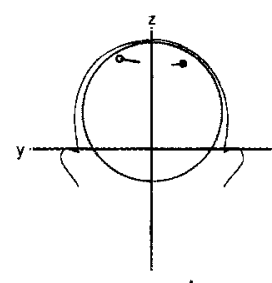

coronal

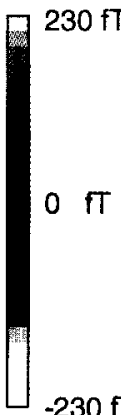

$200 \pi$

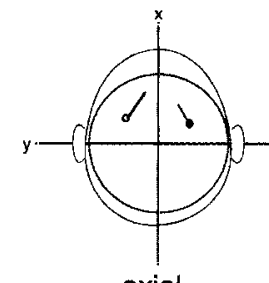

Fig. 3

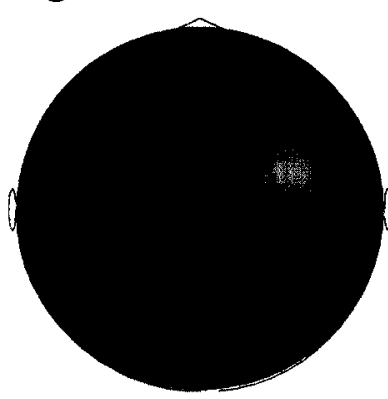

$896 \mathrm{msec}$

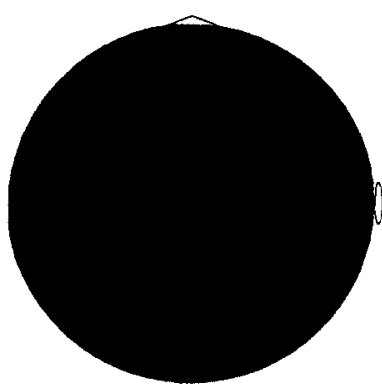

$896 \mathrm{msec}$
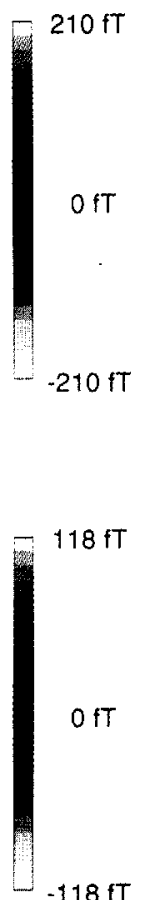

$.118 \mathrm{fT}$
Fig. 2

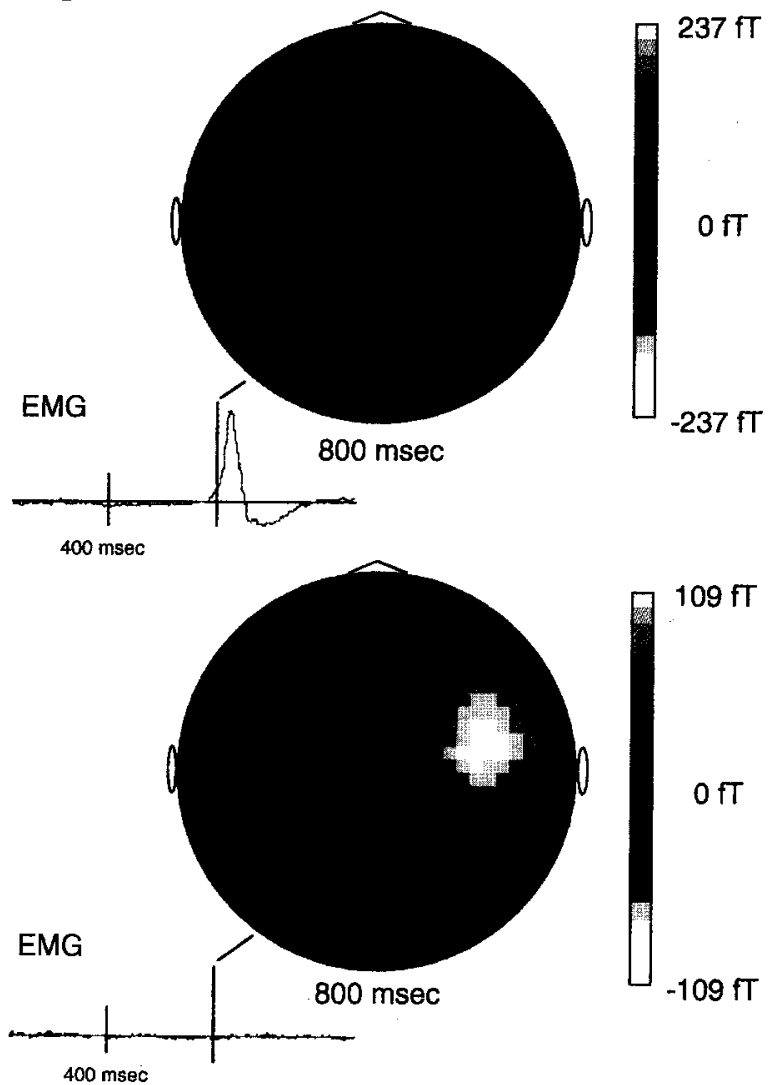

Fig. 4
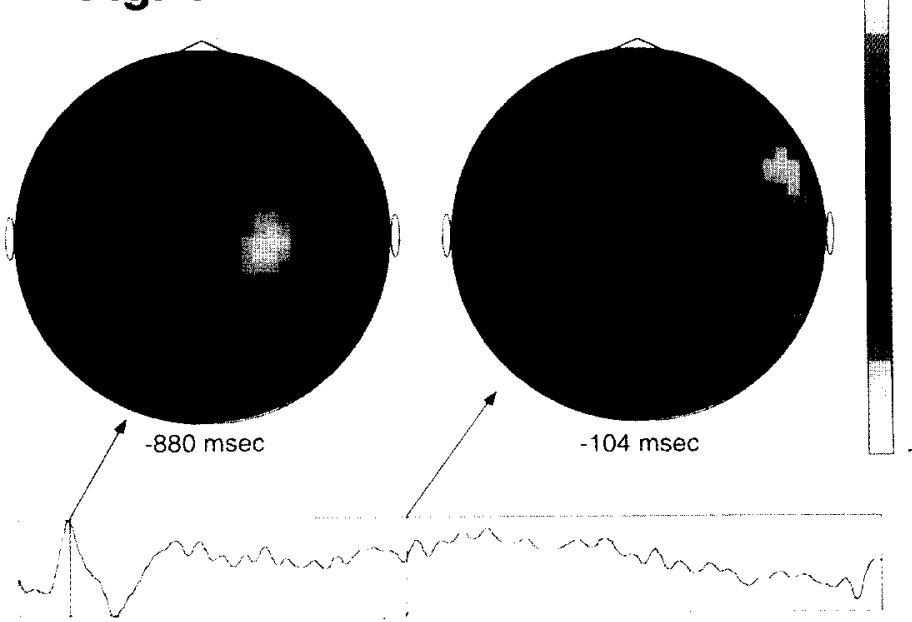
pattern of magnetic fields was found with 'imagined movements' as with executed movements (task 1: Fig. 1; task 2: Fig. 2 upper part). With imagined movements the two dipoles accounted for $72 \%$ of the observed data. Dipole strength with imagined movements was about $50 \%$ of that found with executed movements.

These findings were replicated in a second study with the same subject. Averaged movement onset was $896 \mathrm{~ms}$ after the preceding stimulus in task 2 (flexion at the 'omitted tone'). Patterns of magnetic fields were calculated at this time when subjects initiated the movement (task 2: Fig. 3, upper part) or when they imagined to move (task 3: Fig. 3, lower part). Together with this study an additional control task was used (task 4): subjects were instructed to imagine to hear the 'omitted tone'. Fig. 4 (left side) shows the pattern of the $N 100 \mathrm{~m}$ which was evoked by the presentation of the third tone. Peak latency was $120 \mathrm{~ms}$ (the delay of $20 \mathrm{~ms}$ was caused by transducing the tones via the plastic tubes). $\mathrm{N} 100 \mathrm{~m}$ is generated by surface-todepth neuronal currents in the primary auditory cortices of the two hemispheres [22]. At the time when the subject is assumed to 'imagine the omitted tone' ( $896 \mathrm{~ms}$ after the preceding stimulus or $104 \mathrm{~ms}$ prior to the 'omitted tone', respectively) a field pattern was observed (Fig. 4, right side) which mirrored that of the auditory $\mathrm{N} 100 \mathrm{~m}$ and might reflect depth-to-surface neuronal currents in the primary auditory cortices of the two hemispheres.

Oscillatory spontaneous activity of about $10 \mathrm{~Hz}$ has recently been detected in multi-channel MEG recordings and located in the rolandic area when subjects internally simulated a karate routine $[32,33]$.

\section{Discussion}

Electrophysiological studies consistently report evidence for MI-activity with ISM. Of recent PET- and fMRI-studies, one reported MI-activity with internal simulation of sequential finger movements [15], but others found no MI-activity with imagery of reaching [8] or with internal simulation of sequential finger movements [28].

Present data do not support the concept that higher motor centers (e,g. SMA) are 'disconnected' from lower centers (MI) with ISM. Evidence from EEG studies is indirect. It is based on the assumption that hemispheric asymmetry of brain potentials in $\mathrm{C} 3 / \mathrm{C} 4$ with movement execution is caused by MI-activity which is larger contralateral than ipsilateral to the performing hand (for a review see [20]). But the possibility cannot be excluded that adjacent parts of the cortex, e.g. area 6 , contribute to the hemispheric asymmetry of brain potentials in $\mathrm{C} 3 / \mathrm{C} 4$. Evidence from MEG experiments is more specific. MI-activity has been shown to cause a particular pattern of magnetic fields $[3,4]$. Adjacent parts of the cortex either do not contribute to the surface-recorded MEG-signal (crown of the pre- and the postcentral gyrus) or cause a reversed pattern of magnetic fields due to opposite orientation of the cortical layers (posterior bank of the precentral sulcus and the central sulcus). These results taken together with earlier observations on the mu-rhythm (see above) and recent MEG studies on oscillatory activity by MEG [33] suggest that $\mathrm{MI}$ is active with internal simulation of movements.

In the current MEG study, magnetic fields were smaller in magnitude for ISM than with movement execution. This finding may indicate that the size and/or amount of MI-activity is different between ISM and movement execution. However, imagined movements may be less synchronized than executed movements. Reduced spatio-temporal coherence of MI activity could result in reduction of amplitudes with ISM.

How can these findings be integrated into a concept of functional anatomy? We observed no electromyographic activity with ISM. Others have reported even task-specific patterns of electromyographic activity with ISM $[17,34]$. Decety [6] used magnetic resonance spectroscopy (MRS) and found no evidence for biochemical activation in the target muscle during ISM. What would be an explanation for MI-activity in the absence of electromyographic activity? (1) MI-activity may be inhibitory with ISM. But data do not support this view since the pattern of magnetic fields with ISM as with movement execution is consistent with surface-to-depth current flow in MI which is assumed to be caused by excitation at apical dendritic spines of large pyramidal cells. (2) If we assume excitatory MI-activity with ISM the absence of electromyographic activity (at least at a macroscopic level) is of particular interest. It could be that excitation is blocked at the spinal level, although the mechanism of such blocking action is not known. It has been shown by recent studies that the frontal lobe contains several premotor areas which project to MI and directly to the spinal cord [31]. Frontal lobe activity has recently been shown by EEG-studies when movements had to be inhibited in No-Go trials [23] or when incorrectly primed movements (after a conflicting stimulus set) were suppressed [19]). But Naito and Matsumura [23] found no evidence of frontal lobe activity when subjects were required to 'imagine to go'. The latter finding is in conflict with frontal lobe mechanisms involved in blocking of excitatory MI-activity at the spinal level. (3) Lesion studies in monkeys and observations in humans with brain lesions indicate that $\mathrm{MI}$ is critical in the performance of discrete movements of fingers and other parts of the body (review in [24]). PET studies have shown that MI is activated as much by whole hand movements as by the independent movement of the fingers [5]. Simultaneous movements of two fingers causes even less activation of MI than sequential movements of these fingers with the frequency of movements being kept constant [29]. Thus, to move or not to move individual digits of the hand may be mediated by excitatory MI-activity.

What is the functional meaning of MI-activity with 
ISM? We cannot comment on whether and how MI-activity is related to mental images of the movement during ISM. Psychophysiological analyses and studies in patients with focal lesions are necessary to achieve insight into the functional meaning of MI-activity with ISM.

\section{Acknowledgements}

This work was supported by the Austrian Research Foundation (FWF; P9239-MED).

\section{References}

[1] Beisteiner, R., Höllinger, P., Lindinger, G., Lang, W. and Berthoz, A., Mental representations of movements. Brain potentials associated with imagination of hand movements, Electroencephalogr. Cin. Neurophysiol., 96 (1995) 183-193.

[2] Chatrian, G.E., Petersen, M.C. and Lazarte, J.A., The blocking of the rolandic wicket rhythm and some central changes related to movement, Electroencephalogr. Clin. Neurophysiol., 11 (1959) $497-510$

[3] Cheyne, D., Weinberg, H., Gaetz, W. and Jantzen, K.J., Motor cortex activity and predicting side of movement: neural network and dipole analysis of pre-movement magnetic fields, Neurosci. Lett., 188 (1995) $1-4$.

[4] Cheyne, D., Kristeva R. and Deecke L., Homuncular organization of human motor cortex as indicated by neuromagnetic recordings, Neurosci. Lett., 122 (1991) 17-20.

[5] Colebatch, J.G., Deiber, M.-B., Passingham, R.E., Friston, K.J. and Frackowiak, R.S.J., Regional cerebral blood flow during voluntary arm and hand movements in human subjects, J. Neurophysiol., 65 (1991) 1392-1401.

[6] Decety, J., Do imagined and executed actions share the same neural substrate?, Cogn. Brain Res., 3 (1996) 87-93.

[7] Decety, J. and Ingvar, D.H., Brain structures participating in mental simulation of motor behavior: a neuropsychological interpretation, Acta Psychol., 73 (1990) 13-34.

[8] Decety, J., Perani, D., Jeannerod, M., Bettinardi, V., Tadary, B., Woods, R., Mazziotta, J.C. and Fazio, F., Mapping motor representations with PET, Nature, 371 (1994) 600-602.

[9] Decety, J., Philippon, B. and Ingvar, D.H., rCBF landscapes during motor performance and motor ideation of graphic gesture, Eur. Arch. Psychiat. Neurol. Sci., 238 (1988) 33-38.

[10] Deecke, L., Grözinger, B. and Kornhuber, H.H., Voluntary finger movement in man: cerebral potentials and theory, Biol. Cybern., 23 (1976) 99-119.

[11] Fox, P.T., Pardo, J.V., Petersen, S.E. and Raichle, M.E., Supplementary motor and premotor responses to actual and imagined hand movements with positron emission tomography, Soc. Neurosci. Abstr., 13 (1987) 1433.

[12] Gastaut, H., Etude electrocorticographique de la reactivite des rhythmes rolandiques, Rev. Neurol. (Paris), 87 (1952) 176-182.

[13] Gastaut, H., Naquet, R. and Gastaut, Y., A study of the mu rhythm in subjects lacking one or more limbs, Electroencephalogr. Clin. Neurophysiol., 18 (1965) 720-721.

[14] Graf, M., Niedermeyer, E., Schiemann, J., Uematsu, S. and Long, D.M., Electrocorticography: information derived from intraoperative recordings during seizure surgery, Clin. Electroencephalogr., 15 (1984) 83-91.

[15] Hallett, M., Fieldman, J., Cohen, L.G., Sadato, N. and Pascual-Leone, A., Involvement of primary motor cortex in motor imagery and mental practice, Behav. Brain Sci., 17 (1994) 210.

[16] Ingvar, D.H. and Philipson, L., Distribution of cerebral blood flow in the dominant hemisphere during motor ideation and motor performance, Ann. Neurol., 2 (1977) 230-237.

[17] Jacobsen, E., Electrophysiology of mental activities, Am. J. Physiol., 44 (1932) 677-694.

[18] Klass, D. and Bickford, R.G., Observations on the rolandic arceau rhythm, Electroencephalogr. Clin. Neurophysiol., 9 (1957) 570.

[19] Kopp, B., Rist, F. and Mattler, U., N200 in the flanker task as a neurobehavioral tool for investigating executive control, Psychophysiology, in press.

[20] Lang, W., Höllinger, P., Egkher, A. and Lindinger, G., Functional localization of motor processes in the primary and supplementary motor areas, J. Clin. Neurophysiol., 11 (1994) 397-419.

[21] Lang, W., Zilch, O., Koska, Ch., Lindinger, G. and Deecke, L., Negative cortical DC shifts preceding and accompanying simple and complex sequential movements, Exp. Brain Res., 74 (1989) 99-104.

[22] Näätänen, R. and Picton, T., The N1 wave of the human electric and magnetic response to sound: A review and an analysis of the component structure, Psychophysiology, 24 (1987) 375-425.

[23] Naito, E. and Matsumura, M., Movement-related potentials associated with motor inhibition as determined by use of a stop signal paradigm in humans, Cogn. Brain Res., 2 (1994) 139-146.

[24] Passingham, R., The frontal lobes and voluntary action, Oxford University Press, Oxford, 1993.

[25] Pfurtscheller, G. and Berghold, A., Patterns of cortical activation during planning of voluntary movement, Electroencephalogr. Clin. Neurophysiol., 72 (1989) 250-258.

[26] Rao, S.M., Binder, J.R., Bandettini, P.A., Hammeke, T.A., Yetkin F.Z., Jesmanowicz, A., Lisk, L.M., Morris, G.L., Mueller, W.M., Estkowski, L.D., Wong, E.C., Haughton, V.M. and Hyde, J.S., Functional magnetic resonance imaging of complex human movements, Neurology, 43 (1993) 2311-2318.

[27] Roland, P.E., Larsen, B., Lassen, N.A. and Skinhoj, E., Supplementary motor area and other cortical areas in organization of voluntary movements in man, J. Neurophysiol., 43 (1980) 118-136.

[28] Sanes, J.N., Neurophysiology of preparation, movement and imagery, Behav. Brain Sci., 17 (1994) 221-223.

[29] Shibasaki, H., Sadato, N., Lyshkow, H., Yonekura, Y., Honda, M., Nagamine, T., Suwazono, S., Magata, Y., Ikeda, A., Miyazaki, M., Fukuyama, H., Asato, R. and Konishi, J., Both primary motor cortex and supplementary motor area play an important role in complex finger movement, Brain, 116 (1993) 1387-1398.

[30] Stephan, K.M., Fink, G.R., Frith, C.D. and Frackowiak, R.S.J., Functional anatomy of mental representation of hand movements in healthy subjects, International Union of Physiological Sciences (Glasgow), Abstract 497/P.

[31] Strick, P.L., New insights into motor system connectivity, Mov. Dis., 9, S1 (1994) 11

[32] Tesche, C.D., Imaging cognitive activity with MEG, Presentation at the Department of Medical Informatics, Graz, 1994.

[33] Tesche, C.D., Uusitalo, M.A., IImoniemi, R.J., Huotilainen, M., Kajola, M. and Salonen, O., Signal-space projections of MEG data characterize both distributed and well-localized neuronal sources, Electroencephalogr. Clin. Neurophysiol., in press.

[34] Wehner, T., Vogt, S. and Stadler, M., Task-specific EMG characteristics during mental practice, Psychol. Res., 46 (1984) 369-401. 